\title{
Derechos laborales de mujeres en institutos de Educación Secundaria de Siuna, Costa Caribe Norte de Nicaragua
}

\author{
Iris Concepción Mejía Medrano' \\ Delia Elizabeth Flores Cruz \\ Jacoba Estela Rodríguez Andrade ${ }^{3}$
}

\section{Resumen}

F sta investigación ha analizado los derechos laborales de las mujeres de las instituciones educativas Ede secundaria de la ciudad de Siuna. Se trata de un estudio cualitativo sustentado en un diseño fenomenológico con la finalidad de referir el conocimiento que tienen las mujeres de los derechos laborales, describir los obstáculos que enfrentan, para así proponer acciones que contribuyan al cumplimiento real de sus derechos. Para ello, se suministró la entrevista y la observación a mujeres docentes y mujeres en área administrativas de los Institutos de Educación Secundaria: Roger López Borge, Parroquial San Francisco de Asís y Nueva Esperanza de la ciudad de Siuna. Los principales resultados muestran que los derechos laborales conocidos por las mujeres son: recibir salario por las jornadas trabajadas, pago de horas extras, seguro social, seguro médico, vacaciones, treceavo mes y permisos para asistir a consultas y citas médicas y durante el embarazo derecho a los períodos prenatales y postnatales; y los obstáculos que han enfrentado las mujeres docentes y administrativas, están referidos a la participación en actividades del partido, problemas de salud, cambio de docencia a otros centros, desigualdad en el trato del personal, desconocimiento de las leyes, carencia de equipos y materiales y condiciones de trabajo. En conclusión, las acciones que se proponen están dimensionadas a través de la promoción de la divulgación, investigación, capacitación en material laboral, principalmente en relación a los derechos laborales.

Palabras clave: Derecho al trabajo; conocimiento; obstáculos; acciones propuestas.

\section{Abstract}

This research has analyzed women's labor rights in secondary education institutions in the city of Siuna. This is a qualitative study based on a phenomenological design with the purpose of referring women's knowledge of labor rights, describing the obstacles they face, in order to propose actions that contribute to the real fulfillment of their rights. For this reason, the interview and observation was provided to women teachers and women in administrative areas of the Institutes of Secondary Education: Roger López Borge, Parroquial San Francisco de Asís and Nueva Esperanza of Siuna city. The main results show that the labor rights known by women are: to receive wage for the worked hours, overtime payment, social insurance, health insurance, holidays, thirteenth month and permissions to attend consultation and medical appointments and during the pregnancy the right to prenatal and postnatal periods; And the obstacles faced by women teachers and administrators, are related to participation in party activities, health problems, change of teaching to other centers, inequality in the treatment of staff, lack of knowledge of laws, lack of equipment and materials and working conditions. In conclusion, the actions proposed

\footnotetext{
1 Licenciada en Ciencias Sociales con mención en Desarrollo Local. irismejia94@yahoo.es

2 Licenciada en Ciencias Sociales con mención en Desarrollo Local. deliaelizabethflorescruz@yahoo.es

3 Máster en Educación Intercultural Multilingüe. Asistente de Biblioteca de la Universidad de las Regiones Autónomas de la Costa Caribe Nicaragüense-Recinto Universitario Las Minas. Correo: estela460@hotmail.com
}

Recibido: 03/05/2017 Aprobado: 12/07/2017 
are dimensioned through the promotion of the dissemination, research, training in labor matters, mainly related to labor rights.

Keywords: Right to work; knowledge; Obstacles; Proposed actions.

\section{Introducción}

Esta investigación trata de analizar los derechos laborales de las mujeres de las instituciones educativas de secundaria de la ciudad de Siuna. Existe una preocupación por las organizaciones que defienden los derechos de las mujeres, ya que la violación a los derechos laborales de las mujeres ha sido evidente en las instituciones educativas del país.

Aunque a nivel mundial se han mejorado las condiciones de vida de las mujeres en el mundo en relación con las prohibiciones de la discriminación de género en materia de herencia y ciudadanía, las leyes que garantizan la igualdad en el interior de las familias y políticas que velan por el acceso de la mujer a los servicios públicos de salud y educación, entre otros (ONU, 2010, p. 1). En la Región Autónoma de la Costa Caribe Norte de Nicaragua la participación de las mujeres en los ámbitos laborales ha sido limitada. También, han sido discriminadas por considerarlas del sexo débil y por la falta de toma de decisiones, debido a que en general y culturalmente ha decidido el género masculino (Berrón; 2013, p. 34). En este sentido, es necesario, referir en el conocimiento que tienen sobre el derecho laboral y determinar los obstáculos que enfrentan para el ejercicio de sus derechos laborales en los centros educativos.

\section{Revisión de literatura}

En el contexto nicaragüense, la ley de carrera docente (Ley 114) tiene por objeto establecer las condiciones necesarias que permitan ofrecer al pueblo una educación de calidad y garantizar la estabilidad laboral, capacitación y promoción de los docentes. Sin embargo, a pesar de que se cuenta con esta ley, hoy día son muchos los docentes que desconocen sus derechos laborales (Berrón; 2013, p. 34).

Así lo afirma Silva-Pérez (200o), que una educación de los derechos laborales nos hace más humanos y más valerosos como individuos, grupos y personas. La persona es el centro de los derechos laborales y del Estado de derecho. Es un derecho humano e indispensable para el progreso económico y social. El acceso pleno y en condiciones de igualdad a la educación es un requisito fundamental para la potenciación de la mujer y un instrumento fundamental para lograr los objetivos de igualdad de género, desarrollo y paz.

Beijing (2000), cada vez se reconoce más la necesidad de promulgar leyes específicas y elaborar políticas para reforzar las aptitudes ejecutivas y profesionales de las mujeres, en particular para ayudarlas a dirigir sus propias empresas. Con ese fin, los gobiernos han elaborado políticas, proyectos y programas encaminados a mejorar la educación de las mujeres. Coca (2005), quien afirma que las mujeres capacitadas participan en el mercado laboral con diferentes modalidades, como puede ser el ejercicio de la profesión, la docencia y existe un aumento de mujeres en puestos de conducción de las empresas, las que van construyendo nuevos modelos de comportamiento para superar las limitaciones que las políticas del mercado laboral y las reglas culturales perciben.

Sin embargo, los obstáculos que enfrentan las mujeres en pleno de sus derechos laborales se destacan por: problemas de salud; depresión laboral; discriminación laboral; desigualdades sociales; carencias de materiales y equipos; y condiciones precarias de trabajo. Los problemas de salud están relación con las enfermedades más frecuentes que se presentan en los docentes de Educación Secundaria es: el estrés, la ansiedad, la depresión, la gripe, "burnout" (también llamado "síndrome de estar quemado" (Alemañy-Martínez 2009),

En relación con la depresión laboral se manifiesta en las docentes como un trastorno en el estado de ánimo donde aparecen sentimientos de dolor profundo, ira, frustración y soledad e impiden que la persona continúe con su vida ordinaria de forma normal durante un tiempo prolongado (Gobierno de Canarias, 2010). El estrés laboral expresa Jelinek (2008), la vida del docente se ve afectada a menudo por el estrés laboral que ocasionan las dependencias donde trabajan, por las exigencias laborales, problemas sociales en la escuela, la falta de atención proveniente de sus autoridades superiores con lleva a que el docente se sienta acorralado.

El cambio de las docentes a otros centros surge por la discriminación dentro de las organizaciones se manifiesta de distintas maneras (Anzil, 2009). La 
más común sucede al momento de asignar tareas. La mujer que llega a ocupar uno de esos puestos, por lo general, lo logra habiendo trabajado un periodo extenso dentro de la organización, haciendo carrera dentro de la misma. Así puede demostrar su capacidad y ser tomada en cuenta.

La desigualdad en el trato del personal docente, Barquero-Leiton (2008), afirma que hay discriminación en los equipos de trabajo, cuando estamos ante la presencia de cualquier acto o intención de preferencia, separación o trato desigual a cualquier persona dentro del proceso de formación y/o actuación del equipo de trabajo. Algo importante en los obstáculos que enfrentan las mujeres son la carencia de equipos y materiales para el ejercicio de la docencia según Noriega-García, (2003), quien señala que cada institución educativa tendrá sus propias necesidades, como materiales incluyendo libros suficientes para el alumnado y la tecnología necesaria para sus investigaciones. Así como las condiciones de trabajo que en los centros educativos son precarias, y de modo que la población estudiantil, y principalmente del campo, tienen pocas posibilidades de transformar la realidad que enfrentan día tras día: falta de agua potable y pupitres, techos a punto de derrumbarse, inodoros en mal estado, entre otras (IEEPP, 2011).

\section{Materiales y métodos}

El presente estudio se enmarca en el enfoque cualitativo porque "utiliza la recolección de datos sin medición numérica para descubrir o afinar preguntas de investigación en el proceso de interpretación" (Hernández, Fernández, \& Baptista, 2010, p. 7). En Bisquerra (2012) se afirma que la investigación cualitativa es una actividad sistemática orientada a la comprensión en profundidad de fenómenos educativos y sociales, a la transformación de prácticas y escenarios socioeducativos, a la toma de decisiones y también hacia el descubrimiento y desarrollo de un cuerpo organizado de conocimiento. A partir de las aportaciones de estos expertos, nuestra investigación se centró en el análisis de los derechos laborales de las mujeres de las instituciones educativas de secundaria de la ciudad de Siuna. Por consiguiente, esta investigación tiene un diseño fenomenológico, porque describe las experiencias individuales subjetivas de las participantes, además, que en el enfoque cualitativo el diseño "es el abordaje general que utilizará en el proceso de investigación" (Hernández et al., p. 492).
Las participantes en esta investigación fueron 35 mujeres docentes y administrativas de los institutos de Educación Secundaria: Roger López Borge; Parroquial San Francisco de Asís; y Nueva Esperanza. En este contexto se aplicaron entrevista a mujeres docentes y administrativa y la observación participativa en los institutos de Educación Secundaria. Se destaca que el análisis y procesamiento de la información a través de una matriz y los resultados permitieron la discusión con el abordaje teórico.

\section{Resultados y discusión}

\section{Conocimiento que tienen las mujeres de sus derechos laborales}

La mayoría de las mujeres docentes y administrativas entrevistadas manifestaron que tienen poco conocimiento de sus derechos laborales; sin embargo, expresaron que tienen derecho a un salario por las jornadas trabajadas, a pago de horas extras, seguro social, vacaciones cada seis meses, treceavo mes conocido como el aguinaldo y permisos para asistir a consultas y citas médicas, y durante el embarazo comentaron que tienen derecho a los periodos prenatales y postnatales. En las entrevistas las docentes manifestaron que los salarios están en dependencia del tipo de cargo y títulos que poseen y de acuerdo a ello se les garantiza el tipo de plaza, ya sea en Educación Primaria o Educación Secundaria.

El salario básico de Educación Secundaria es de $C \$ 6,400$; pero, cuando las docentes poseen otros títulos son reconocidos por el Ministerio de Educación. Por el diploma de Educación Secundaria y diplomados les reconocen $C \$ 100$, por títulos que las acredita como Licenciadas en Educación Superior $C \$ 200$ córdobas y por el título de Maestras de Educación Secundaria (Magisterio) les reconocen $C \$ 300$. De igual manera se les reconoce la antigüedad y zonaje.

Lo antes mencionado coincide con lo que dice el Código del trabajo (1996), en su artículo 84, salario ordinario es el que se devenga durante la jornada ordinaria, en el que están comprendidos el salario básico. También se relaciona con lo que se argumenta en el Reglamento de la ley de carrera docente (1991), en su artículo 75, el monto total del salario del docente será el salario básico, más el escalafón, más el zonaje establecido. 
Al referirse a pagos de horas extras, una funcionaria opinó:

Aquí los derechos se nos violentan, no podemos decir en nuestras casas a qué hora se llega ni se sale, porque a la hora que cualquier funcionario quiera realizar alguna actividad populista debemos acompañarlo sin oposición alguna y uno lo hace porque no hay otro trabajo (Entrevista aplicada 21/o8/2015).

Lo expuesto anteriormente no se relaciona con lo del Código del trabajo (1996), ley $\mathrm{N}^{\circ} 185$ que en su artículo 62 señala que las horas extraordinarias y las que labore el trabajador en su día de descanso o compensatorio por cualquier causa, se pagarán un cien por ciento más de lo estipulado para la jornada normal respectiva.

Del seguro social, una docente señaló:

Tanto que nos esforzamos por trabajar por la educación, somos los que se les paga menos y son los que tienen menos beneficios al momento de tener riesgos. En la jubilación con lo que te da la prestación social no se vive y no se compensa con lo que una retribuye (Entrevista aplicada 21/08/2015).

Lo anterior se relaciona con lo que dice Bolaños (2008), jurídicamente el INSS no está obligado a pagar pensiones de jubilación del 100\% del salario a los docentes que califiquen el derecho de gozar de la pensión de vejez, por ejemplo. El INSS calculará la pensión en base al número de semanas que haya cotizado el docente conforme las disposiciones de seguridad social, siempre y cuando el interesado tenga los años de servicios exigidos y la edad establecida.

Del seguro médico, una docente señaló muy inconforme lo siguiente:

Nos quitan tanto dinero estas instituciones y prácticamente nos ven como algo que no tiene valor, como si nosotros no aportamos para recibir la mejor atención". Hay parejas de jóvenes médicos que tienen sus clínicas y atienden mejor, estos tienen hasta laboratorios para realizar sus exámenes (Entrevista aplicada 21/08/2015).
Lo mencionado anteriormente se relaciona con lo que opina Bolaños (2008), al expresar que las empresas médicas derivan su nombre del llamado "Modelo de Salud Previsional", que fue creado por el INSS para brindar atención médica a los asegurados que pagan los Seguros de EnfermedadMaternidad y riesgos profesionales. Con respecto a las vacaciones, una docente opina: "Es bueno siempre tener sus vacaciones y es saludable para la institución que su personal esté descansado para desarrollar con más energía los procesos académicos" (Entrevista aplicada 21/o8/2015).

Lo planteado anteriormente no coincide con lo establecido en el Reglamento de la ley de carrera docente (1991), que en su artículo 96, afirma que los docentes gozarán de dos meses de descanso anual, según calendario escolar. Derecho al treceavo mes: En sus entrevistas las mujeres manifestaron que cada fin de año por derecho les corresponde el treceavo mes conocido como aguinaldo. Refieren que el aguinaldo es el pago del salario total devengado, sin deducción alguna.

Manifestaron que este es entregado a finales del mes de noviembre por decisión del Gobierno de Reconciliación y Unidad Nacional, aunque según lo establecido en el Código del Trabajo se debe entregar en los primeros diez días del mes de diciembre. El Código del trabajo (1996), en su artículo 94, el salario adicional anual o décimo tercer mes se pagará conforme al último mes de salario recibido, salvo cuando se devengare salario por comisiones, obra, al destajo y cualquier otra modalidad compleja; en estos casos se pagará conforme el salario más alto recibido durante los últimos seis meses. También en su artículo 95 el décimo-tercer mes deberá ser pagado dentro de los primeros diez días del mes de diciembre de cada año, o dentro de los primeros diez días después de terminado el contrato de trabajo.

Derecho a permisos para asistir a consultas y citas médicas: Las docentes y las mujeres del personal administrativo comentaron que en sus centros de Educación Secundaria tienen el permiso para asistir a las consultas médicas a la Clínica Previsional del municipio cuando estas se sienten mal de salud o para llevar a sus hijos e hijas. De igual manera, tienen el permiso para asistir a citas médicas a diferentes hospitales de la Ciudad de Managua a los cuales son remitidas por los médicos en los casos en los cuales requieren exámenes o estudios en otras especialidades y con mayor rigurosidad. 
También se relaciona con lo que dice el Reglamento de la Ley de Carrera Docente (1991), en el artículo 94, se entiende por permiso la autorización que le hace el Ministerio de Educación a un docente para ausentarse de sus labores temporalmente por las razones siguientes: con goce de salario, por subsidio debidamente extendido por el sistema nacional único de salud.

Derecho a períodos prenatales y postnatales: Las docentes y mujeres del personal administrativo de los Institutos de Educación Secundaria expresaron que cuando van a dar a luz a sus hijos e hijas tienen derecho al reposo durante un mes antes del parto y a dos meses posteriores. Lo antes mencionado se coincide con lo la Constitución Política (2014), en su artículo 74, el Estado otorga protección especial al proceso de reproducción humana. La mujer tendrá protección especial durante el embarazo y gozará de licencia con remuneración salarial y adecuada de seguridad social.

\section{Obstáculos que enfrentan las mujeres en el ejercicio de sus derechos laborales}

Problemas de salud: Se encontró que las mujeres padecen muchos problemas de salud, entre los que mencionaron: el chikungunya, lo que dificulta poder dar cumplimiento a su tarea docente. Comentaron que, por estar enfermas, es un atraso para ellas y para sus estudiantes, porque tienen que buscar a docentes sustitutas para que puedan desarrollar los contenidos planificados en la programación. Lo anterior planteado se relaciona con lo de Alemañy Martínez (2009), son enfermedades más frecuentes que se presentan en los docentes de Educación Secundaria es: el estrés, la ansiedad, la depresión, la gripe, "burnout" (también llamado "síndrome de estar quemado).

Depresión laboral: Uno de los problemas de salud, que padecen las docentes, el principal obstáculo que más les afecta es la depresión laboral, la que se presenta cuando se sienten agobiadas de problemas que se generan en la institución, debido al mal comportamiento de sus estudiantes, por problemas de indisciplina, de dificultades de aprendizaje de los mismos que afectan el rendimiento académico, razones que los llevan hasta un punto que sienten que no son eficientes en su labor de docencia.

Lo anterior se relaciona con lo que dice el Gobierno de Canarias (2010), la depresión es un trastorno en el estado de ánimo donde aparecen sentimientos de dolor profundo, ira, frustración y soledad e impiden que la persona continúe con su vida ordinaria de forma normal durante un tiempo prolongado.

Estrés laboral. Una docente expresa: "En algunas ocasiones padecemos de estrés por indisciplina de los estudiantes, desinterés en las aulas de clases y algunas actitudes negativas de sus padres" (Entrevista aplicada 21/o8/2015). Lo planteado anteriormente se relaciona con lo que expresa Jelinek (2008), la vida del docente se ve afectada a menudo por el estrés laboral que ocasionan las dependencias donde trabajan, por las exigencias laborales, problemas sociales en la escuela, la falta de atención proveniente de sus autoridades superiores conlleva a que el docente se sienta acorralado

El cambio de la docencia a otros centros. Al respecto un docente expresa: "Si me van ubicar está bien, pero deberían explicarme el porqué del cambio y cuál es la evaluación que respalda el traslado, siento que solamente somos manejados como objetos y somos docentes que tenemos que respetarnos..." (Entrevista aplicada 21/08/2015). Lo planteado anteriormente se relaciona con lo que expresa Anzil (2009), la discriminación dentro de las organizaciones se manifiesta de distintas maneras. La más común sucede al momento de asignar tareas. La mujer que llega a ocupar uno de esos puestos, por lo general, lo logra habiendo trabajado un periodo extenso dentro de la organización, haciendo carrera dentro de la misma. Así puede demostrar su capacidad y ser tomada en cuenta.

La desigualdad en el trato del personal docente: Las preferencias parentales o de amistad en algunos cargos en los institutos del área urbana del municipio de Siuna, es evidente, manifestaron que los familiares de las directoras o amigas de las mismas tienen privilegios, así como el personal que cuenta con un cargo en los centros de Educación Secundaria.

Lo antes mencionado coincide con lo que dice Barquero Leiton (2008), quien afirma que hay discriminación en los equipos de trabajo, cuando estamos ante la presencia de cualquier acto o intención de preferencia, separación o trato desigual a cualquier persona dentro del proceso de formación y/o actuación del equipo de trabajo. 
Desconocimiento de las leyes: En las entrevistas aplicadas a las docentes y a las mujeres del personal administrativo manifestaron que a pesar que conocen algunos derechos como trabajadoras, desconocen a profundidad los artículos que contienen cada una de las leyes que protegen a las mujeres. Mencionaron que conocen la existencia del código del trabajo, la Constitución Política de la República de Nicaragua, la Ley 779 , pero consideran que no poseen los conocimientos necesarios para defender sus derechos.

Carencia de equipos y materiales: Las docentes dijeron que tienen problemas con los marcadores y borradores que son entregados para impartir sus clases. Les hacen entrega de cuadernos dependiendo de las asignaturas que imparten, les facilitan lapiceros utilizados para la planificación docente, pero cuando se les agota tienen que hacer las compras incurriendo en gastos. También expresaron que carecen de suficientes libros de textos que puedan ser utilizados para realizar trabajos en grupos en las aulas de clases. Lo antes mencionado concuerda con lo que expresan Noriega García, (2003), quien señala que cada institución educativa tendrá sus propias necesidades, como materiales incluyendo libros suficientes para el alumnado y la tecnología necesaria para sus investigaciones.

Condiciones de trabajo en las aulas de clases: Otro de los problemas encontrados es que en los institutos sabatinos "Nueva Esperanza" y "Roger López Borge", las condiciones higiénicas sanitarias son críticas, porque se encuentran en mal estado, lo que afecta a la comunidad educativa. Por otro lado, las docentes del Instituto Parroquial expresaron que tienen problemas de iluminación, incluso manifestaron que, en la época de invierno, cuando hay fuertes lluvias tienen que suspender las clases debido a la oscuridad que se refleja en las aulas.

Lo ante mencionado coincide con lo que expresa el Instituto de Estudios Estratégicos y Políticas Públicas, (2011), de modo que la población estudiantil, y principalmente del campo, tienen pocas posibilidades de transformar la realidad que enfrentan día A día: falta de agua potable y pupitres, techos a punto de derrumbarse, inodoros en mal estado, entre otras.

\section{Contribución al cumplimiento de los derechos laborales de las mujeres}

$\mathrm{Al}$ respecto una docente refirió:
Lo más idóneo es conocer los derechos que tenemos como trabajadoras, debe ser un interés del sindicato, que las mujeres docentes y administrativas tengamos conocimientos claros sobre derechos laborales y así poder reclamar y defendernos en el momento que sean violentados (Entrevista aplicada 21/o8/2015).

Lo planteado anteriormente coincide con lo que expresa el Ministerio del Trabajo, Empleo y Previsión Social y la Dirección General de Empleo, (2013), quien afirma que en Nicaragua se deben continuar haciendo programas radiales con el propósito de divulgar y conocer los derechos laborales.

Las entrevistadas manifestaron que se deben implementar el desarrollo de capacitaciones referidas a los derechos laborales para evidenciar y manejar en tres dinámicas los procesos, el primero que debe orientarse a deberes como funcionarias, el segundo de derechos y el tercero a procedimientos que deben seguirse para dar salida a cada una de las acciones y los problemas que se enfrentan laboralmente. Lo anterior se relaciona con lo que expresa Coca (2005), quien afirma que las mujeres capacitadas participan en el mercado laboral con diferentes modalidades, como puede ser el ejercicio de la profesión, la docencia, entre otras.

\section{Conclusiones}

Según los resultados obtenidos en la presente investigación llegamos a las siguientes conclusiones:

En los Institutos "Roger López Borge", instituto Parroquial "San Francisco de Asís" e instituto "Nueva Esperanza", mujeres del personal docente como administrativo, tienen conocimientos de sus derechos laborales, entre ellos: recibir salario por las jornadas trabajadas, pago de horas extras, seguro social, seguro médico, vacaciones, treceavo mes, permisos para asistir a consultas, citas médicas y derecho a períodos prenatales y postnatales, pero es necesario que conozcan en qué artículos de los instrumentos jurídicos están contenidos los mismos.

Los obstáculos identificados y que enfrentan las mujeres docentes y administrativas, se refieren a la participación en actividades partidarias, problemas de salud, cambio de docencia a otros centros, desigualdad en el trato, desconocimiento 
de las leyes, carencia de equipos y materiales y condiciones de trabajo.

Las acciones que se proponen están dimensionadas a través de la promoción de la divulgación, investigación y capacitación en legislación laboral, acciones e incidencia, auditoría con perspectivas de género y un programa de formación establecidos en los instrumentos jurídicos vigentes.

\section{Bibliografía}

Alemañy Martínez, C. (2009). La docencia: enfermedades frecuentes de esta profesión. Recuperado de: http://www.eumed.net/rev/ced/o2/cam6. htm

Anzil, F. (2009). La discriminación de género. Recuperado de: www.equidad.org.mx

Asamblea Nacional de Nicaragua (1990). Ley de carrera Docente. Ley 114. Managua: La Gaceta

Asamblea Nacional. (2014). Constitución Política de la República de Nicaragua.

Asamblea Nacional. (1991). Reglamento de ley de carrera docente. Acuerdo Ministerial No. 38, aprobado el o3 de Juniojunio de 1991. Publicada en La Gaceta Diario Oficial No 169 del 10 de Septiembreseptiembre de 1991. Nicaragua.

Asamblea Nacional. (1996). Ley No. 185. Código del trabajo. Publicada en La Gaceta Diario Oficial No. 205, del 30 de octubre de 1996. Nicaragua.

Asamblea Nacional. (2008). Ley $\mathrm{N}^{\circ}$. 648. Ley de igualdad de derechos y oportunidades. Publicada en La Gaceta Diario Oficial No.15o, del 14 de febrero del 2008. Nicaragua.

Barquero-Leiton, M. (2008). Manual de derechos laborales para las mujeres.

Berron, G. (2013). Vieios y Nuevos Dilemas Para la Farticipacion Social en los Procesos de Integracion Sudamericanos. Cadernos Prolam/ USP, 12(23), 42-55.
Beijing. (200o). La mujer la economía. Recuperado de http://www.un.org/spanish/conferences/ Beijing/fs6.htm

Bisquerra, R., (2012). Metodología de la Investigación Educativa. Madrid: La Muralla.

Bolaños, L. M. (2008). Diagnóstico de la violencia intrafamiliar y sexual de Nicaragua. Recuperado de http://www.policia.gob.ni/cedoc/sector/ dgt/Diagnostico_Violencia_Intrafamiliar.pd

Chávez, K. (2013). Nicaragua mundialmente reconocida en equidad de género y empoderamiento de las mujeres. Recuperado de http:// www.el19digital.com/articulos/ver/titulo:14243-nicaragua-mundialmente- reconocida-en-equidad-de-genero-y-empoderamiento-de-las-mujeres.

Coca, H. (2005). El papel de la mujer. Recuperado de www.gestiopolis.com/ Canales4/rrhh/mujerpapel.htm

Comisión de Asuntos Laborales y Gremiales de Nicaragua (2015). Foro sobre prácticas de género en las relaciones laborales. Recuperado de: http://www.asamblea.gob.ni/337916/ realizan-foro-sobre-practicas-de-generoen-las-relaciones-laborales/

Gobierno de Canarias España (2010). Depresión en el trabajo. Recuperado de http://www.gobiernodecanarias.org/sanidad/scs/susalud/ susalud.htm

Hernández, R., Fernández, C., \& Baptista, M. (2010). Metodología de la Investigación. México: McGraw-Hill.

Instituto de Estudios Estratégicos y Políticas Públicas (2011). Falta de infraestructura escolar afectacalidad en la educación. Recuperado de http://www.ieepp.org/index.php/falta-de-infraestructura-escolar-afecta-calidaden-la-educacion/

Jelinek, R. (2008). Todos los seres humanos nacen libres e iguales en dignidad de derecho. Recuperado de http://www.confe.org.mx/ congreso/archivos/2008/3_nov/conferencia/ raquel_jeli nek_derechos_humanos_y_vida_ cotidiana.pdf. 
Ley No. 114. Ley de Carrera Docente. Publicada en La Gaceta No. 225 del 22 de Noviembre de 1990.

Ministerio del Trabajo, Empleo y Previsión Social y la Dirección General de Empleo (2013). Los derechos fundamentales y laborales en programa radial. Recuperado de http://empleo.gob.bo/ index.php?option=com_content\&view $=$ article\&id=58:los- derechos-fundamentales-y-laborales-en-programa-radial

Noriega García, J. L. (2003). Calidad de la educación. Recuperado de http://www.monografias. com/trwabajos24/calidad-educativa/calidad-educativa.shtml\#ixzzzsixqtDSo
ONU. (2010). Convención Sobre la Eliminación de todas las Formas de Discriminación Contra la Mujer. Panamá: UNICEF.

Silva Pérez, A. E. (2000). Agenda de los derechos laborales. Recuperado de www.mec.org.ni/ wp.../o5/Diagramacion-Marielena-ADL-ABfull.2.pdf el pdf agenda de los derechos laborales de la mujer. 